\title{
The Impact of Intraoperative Hypothermia on Blood Loss and Allogenic Blood Transfusion in Total Knee and Hip Arthroplasty: A Retrospective Study
}

\author{
Pin Pan $\mathbb{D}$, ${ }^{1,2}$ Kai Song $\mathbb{D}$, , ${ }^{1,2}$ Yao Yao $\mathbb{D}$, ${ }^{1,2}$ Tao Jiang $\mathbb{D}$, ${ }^{1,2}$ and Qing Jiang $\mathbb{D}^{1,2}$ \\ ${ }^{1}$ Department of Sports Medicine and Adult Reconstructive Surgery, Drum Tower Hospital, School of Medicine, Nanjing University, \\ 321 Zhongshan Road, Nanjing, 210008 Jiangsu, China \\ ${ }^{2}$ Laboratory for Bone and Joint Disease, Model Animal Research Center (MARC), Nanjing University, Nanjing, \\ 210093 Jiangsu, China
}

Correspondence should be addressed to Qing Jiang; qingj@nju.edu.cn

Received 24 November 2019; Revised 21 March 2020; Accepted 27 April 2020; Published 5 May 2020

Academic Editor: Alberto Grassi

Copyright (C) 2020 Pin Pan et al. This is an open access article distributed under the Creative Commons Attribution License, which permits unrestricted use, distribution, and reproduction in any medium, provided the original work is properly cited.

Background. Total joint arthroplasty (TJA) usually leads to substantial blood loss, which may cause allogenic blood transfusion. Hypothermia occurring during operation has been reported to increase blood loss and transfusion rates in nonorthopedic cohorts. However, the relationship between intraoperative hypothermia and blood loss remains controversial in patients undergoing orthopedic surgeries. The aims of this study were to investigate the incidence of hypothermia and identify the impact of intraoperative body temperature and hypothermia on blood loss and transfusion rates in total knee and hip arthroplasty (TKA and THA, respectively). Methods. This retrospective study enrolled 616 consecutive patients, who underwent primary unilateral TKA or THA at our institution during the period from April 2012 to July 2014. The occurrence of a temperature below $36^{\circ} \mathrm{C}$ during the operation was documented to identify the incidence of hypothermia. Univariate analysis was performed to find the risk factors for hypothermia. Multiple regression analysis and multivariate logistic regression analysis were employed to explore the association of intraoperative temperature and hypothermia with intraoperative blood loss and perioperative blood transfusion. Results. The incidence of intraoperative hypothermia was $13.5 \%, 14.0 \%$, and $13.1 \%$ in TJA, TKA, and THA, respectively. Intraoperative temperature $(P=0.045, P=0.006)$ and hypothermia $(P=0.042, P<0.001)$ were associated with intraoperative blood loss and perioperative transfusion in TKA. Intraoperative temperature $(P=0.002)$ was negatively related to the amount of blood loss, and hypothermia $(P=0.031)$ was the independent risk factor for transfusion in THA. Conclusion. Intraoperative hypothermia is associated with increased blood loss and transfusion rates in TJA. Efforts should be made to maintain normothermia during operation in these patients.

\section{Introduction}

Total joint arthroplasty (TJA) can cause substantial blood loss, which may lead to the requirement of allogeneic blood transfusion. Transfusion is associated with potential risks including transmission of infectious diseases, immunological reaction, cardiopulmonary complications, and increased length of stay $[1,2]$. Therefore, the value of identifying modifiable risk factors for blood loss and transfusion is well recognized.
Intraoperative hypothermia has proven to be associated with higher rates of blood transfusion in nonorthopedic cohorts [3]. The relationship between intraoperative hypothermia and blood loss remains controversial in patients undergoing orthopedic surgeries [4]. Maintaining body temperature during spinal surgeries under general anesthesia reduces blood loss and transfusion rate. However, other studies did not find the association of hypothermia with blood loss in shoulder arthroplasty [5], pediatric posterior spinal fusion [6], and open lumbar spine surgery [7]. Hypothermia, 
which is defined as a core body temperature below $36^{\circ} \mathrm{C}$ [8], is a common event during operations due to the impacts of anesthetics and prolonged skin exposure to the cool environment of the operation room. It is also related to thermal discomfort, increased risk of cardiac morbidity, higher rates of surgical site infections, prolonged effects of anesthetics, and increased length of hospital stay $[3,9,10]$.

Patients undergoing TJA are at high risk of hypothermia during surgery, especially in the setting of general anesthesia. Previous studies reported hypothermia rates of $11.2 \%-34 \%$ for total knee arthroplasty (TKA) and $13.2 \%-43.9 \%$ for total hip arthroplasty (THA) [11-13]. Hypothermia has proven to be associated with increased rates of readmission and deep infection [11]. However, whether hypothermia is related to increased blood loss and higher transfusion rates remains controversial in TJA $[13,14]$.

Therefore, we conducted this study in TKA and THA cohorts to determine the prevalence of intraoperative hypothermia and to explore the impact of intraoperative temperature and hypothermia on blood loss and transfusion rates.

\section{Material and Methods}

This study was approved by the Institutional Review Board (reference number: 2016-063-02). Patients who underwent primary unilateral TKA or THA consecutively at our institution between April 2012 and July 2014 were included in this retrospective study. If patients received staged TJA during the research period, only the first procedure was included. We excluded patients with coagulation disorders or on anticoagulants before surgery. Patient demographic and clinical data was collected from medical records, including age, gender, body mass index (BMI), preexisting illnesses (diabetes, hypertension, and malignancy), primary diagnosis, and operation time. The data of preoperative laboratory examinations, such as hemoglobin (Hb) concentration, blood platelet count (PLT), plasma prothrombin time (PT), international normalized ratio (INR), and activated partial thromboplastin time (APTT), was also documented.

Surgeries were performed by four experienced surgeons under general anesthesia. A tourniquet was used in all patients undergoing TKA. The tourniquet was inflated before skin incision and deflated after cementing the prosthesis. All patients received propofol-based total intravenous anesthesia. Continuous infusion of propofol, remifentanil, and cisatracurium was employed during the surgery. Ventilation was controlled to maintain end-tidal $\mathrm{CO}_{2}$ at $30-35 \mathrm{mmHg}$. All patients had normal body temperature before anesthesia. Active warming devices were not routinely used. If a temperature lower than $36^{\circ} \mathrm{C}$ was detected during the surgery, forced air warming would be used to maintain the body temperature. Body temperature was recorded via esophageal probe every 5 minutes during the surgery. In this study, the term "intraoperative temperature" used in the analysis was calculated by taking the average of temperature readings during the surgery, and the term "hypothermia" described a body temperature nadir lower than $36^{\circ} \mathrm{C}$ during the surgery. Intraoperative blood loss was calculated by the volume of blood in the suction canister plus the estimated amount of blood in the surgical sponges. The amount of blood in the suction canister was estimated by the total volume of fluid in the canister minus the volume of irrigation solution during the surgery. The surgical sponges were weighed before and after surgical use. The estimated amount of blood in the sponges was determined by calculating the weight difference before and after use. None of the patients received transfusion preoperatively. During the operation, blood transfusion was performed if the level of hemoglobin $(\mathrm{Hb})$ decreased lower than $9.0 \mathrm{~g} / \mathrm{dl}$. After the surgery, patients with a hemoglobin level of less than $8.0 \mathrm{~g} / \mathrm{dl}$ or developing symptomatic anemia with a hemoglobin level of less than $9.0 \mathrm{~g} / \mathrm{dl}$ would receive transfusion. The events of perioperative transfusion were recorded. Drainage tubes were routinely placed in these patients and removed within 48 hours postoperatively. All patients received recommended thromboembolism prophylaxis regimen (rivaroxaban or low-molecular-weight heparin) after surgery. Tranexamic acid was not used routinely in these patients perioperatively.

Statistical analysis was conducted using Stata, version 12.0 (StataCorp LP, College Station, TX). To explore the risk factors for intraoperative hypothermia, we compared age, gender, BMI, preexisting illnesses, primary diagnosis, operation time, laboratory data, intraoperative blood loss, and perioperative blood transfusion between the hypothermia group and the nonhypothermia group. Qualitative variables were analyzed using a chi-square test, and quantitative variables were analyzed using a $t$-test. Multiple regression analysis was employed to determine whether intraoperative temperature and hypothermia are associated with blood loss after adjustment for age, gender, BMI, preexisting illnesses, diagnosis, preoperative laboratory data, and operation time. Similarly, we performed multivariate logistic regression analysis to explore whether intraoperative temperature and hypothermia affect perioperative transfusion rates independently.

\section{Results}

A total of 616 patients were enrolled in this study with a mean age of $65.9 \pm 11.8$ years (range 20-93 years). Two hundred and seventy-two patients received TKA, and 344 patients underwent THA. There were 426 females and 190 males. The mean intraoperative temperature was $36.4 \pm 0.2^{\circ} \mathrm{C}$ (range 35.8$36.8^{\circ} \mathrm{C}$ ). Eighty-three patients (13.5\%) experienced an event of hypothermia during the surgery. The mean amount of intraoperative blood loss was $208.7 \pm 131.3 \mathrm{ml}$ (range $40-1050 \mathrm{ml})$. There were 94 patients (15.3\%) receiving allogeneic transfusion during the perioperative period. The characteristics of these patients are summarized in Table 1.

There were 222 females and 50 males undergoing TKA with a mean age of $68.0 \pm 7.3$ years (range $40-86$ years). They were diagnosed with osteoarthritis or rheumatoid arthritis. The mean intraoperative temperature was $36.4 \pm 0.2^{\circ} \mathrm{C}$ (range $35.8-36.8^{\circ} \mathrm{C}$ ). The incidence of intraoperative hypothermia was $14.0 \%(n=38)$. The mean amount of intraoperative blood loss was $196.0 \pm 103.2 \mathrm{ml}$ (range $50-600 \mathrm{ml}$ ). Forty-eight patients (17.6\%) received perioperative transfusion. According to the univariate analysis, we determined 
Table 1: Patient's demographic and clinical characteristics.

\begin{tabular}{|c|c|c|c|}
\hline Characteristics & TJA $(n=616)$ & TKA $(\mathrm{n}=272)$ & THA $(\mathrm{n}=344)$ \\
\hline Age $($ years $($ mean $\pm S D))$ & $65.9 \pm 11.8$ & $68.0 \pm 7.3$ & $64.2 \pm 14.1$ \\
\hline \multicolumn{4}{|l|}{ Gender } \\
\hline Male (\%) & $190(30.8)$ & $50(18.4)$ & $140(40.7)$ \\
\hline Female (\%) & $426(69.2)$ & $222(81.6)$ & $204(59.3)$ \\
\hline BMI $\left(\mathrm{kg} / \mathrm{m}^{2}(\right.$ mean $\left.\pm \mathrm{SD})\right)$ & $24.8 \pm 3.9$ & $26.2 \pm 3.7$ & $23.6 \pm 3.6$ \\
\hline Diabetes (\%) & $85(13.8)$ & $50(18.4)$ & $35(10.2)$ \\
\hline Hypertension (\%) & $237(38.5)$ & $122(44.9)$ & $115(33.4)$ \\
\hline Malignancy (\%) & $15(2.4)$ & $8(2.9)$ & $7(2.0)$ \\
\hline \multicolumn{4}{|l|}{ Diagnosis } \\
\hline OA (\%) & $299(48.5)$ & $246(90.4)$ & $53(15.4)$ \\
\hline RA (\%) & $33(5.4)$ & $26(9.6)$ & $7(2.0)$ \\
\hline Femoral neck fracture (\%) & $147(23.9)$ & - & $147(42.7)$ \\
\hline ONFH (\%) & $79(12.8)$ & - & $79(23.0)$ \\
\hline $\mathrm{DDH}(\%)$ & $35(5.7)$ & - & $35(10.2)$ \\
\hline AS (\%) & $23(3.7)$ & - & $23(6.7)$ \\
\hline Operation time $($ min $($ mean $\pm S D))$ & $111.9 \pm 28.0$ & $124.1 \pm 23.4$ & $102.2 \pm 27.6$ \\
\hline $\mathrm{PT}(\mathrm{s}($ mean $\pm \mathrm{SD}))$ & $11.5 \pm 1.0$ & $11.3 \pm 0.9$ & $11.6 \pm 1.0$ \\
\hline INR $($ mean \pm SD) & $1.01 \pm 0.08$ & $0.99 \pm 0.08$ & $1.01 \pm 0.09$ \\
\hline $\operatorname{APTT}(\mathrm{s}($ mean $\pm \mathrm{SD}))$ & $26.9 \pm 4.5$ & $26.0 \pm 3.8$ & $27.6 \pm 4.9$ \\
\hline $\mathrm{Hb}(\mathrm{g} / \mathrm{l}($ mean $\pm \mathrm{SD}))$ & $127.7 \pm 14.5$ & $126.2 \pm 13.4$ & $129.0 \pm 15.2$ \\
\hline $\operatorname{PLT}\left(10^{9} / 1(\right.$ mean $\left.\pm \mathrm{SD})\right)$ & $203.2 \pm 66.6$ & $206.8 \pm 69.2$ & $200.3 \pm 64.5$ \\
\hline Intraoperative temperature $\left({ }^{\circ} \mathrm{C}(\right.$ mean $\left.\pm \mathrm{SD})\right)$ & $36.4 \pm 0.2$ & $36.4 \pm 0.2$ & $36.3 \pm 0.1$ \\
\hline Hypothermia (\%) & $83(13.5)$ & $38(14.0)$ & $45(13.1)$ \\
\hline Intraoperative blood loss $(\mathrm{ml}($ mean $\pm \mathrm{SD}))$ & $208.7 \pm 131.3$ & $196.0 \pm 103.2$ & $218.7 \pm 149.3$ \\
\hline Allogenic blood transfusion (\%) & $94(15.3)$ & $48(17.6)$ & $46(13.4)$ \\
\hline
\end{tabular}

TJA: total joint arthroplasty; TKA: total knee arthroplasty; THA: total hip arthroplasty; SD: standard deviation; BMI: body mass index; OA: osteoarthritis; RA: rheumatoid arthritis; ONFH: osteonecrosis of the femur head; DDH: developmental dysplasia of the hip; AS: ankylosing spondylitis; PT: prothrombin time; INR: international normalized ratio; APTT: activated partial thromboplastin time; Hb: hemoglobin; PLT: platelet count.

the association of intraoperative blood loss $(P=0.005)$ and perioperative transfusion $(P<0.001)$ with hypothermia in the TKA group (Table 2). Both intraoperative temperature $(P=0.045)$ and hypothermia $(P=0.042)$ were associated with intraoperative blood loss after adjustment using multiple regression analysis (Table 3). Subsequently, we conducted multivariate logistic regression analysis and found that intraoperative temperature $(P=0.006, \mathrm{OR}=0.080)$ and hypothermia $(P<0.001, \mathrm{OR}=4.468)$ were independently related to transfusion rates (Table 3 ).

A total of 344 patients received THA for femoral neck fracture, osteonecrosis of the femoral head, primary osteoarthritis, developmental dysplasia of the hip, rheumatoid arthritis, or ankylosing spondylitis. There were 204 females and 140 males with a mean age of $64.2 \pm 14.1$ years (range $20-93$ years). The mean intraoperative temperature was $36.3 \pm 0.1^{\circ} \mathrm{C}$ (range 36.0-36. $\left.7^{\circ} \mathrm{C}\right)$. Forty-five patients $(13.1 \%)$ experienced an event of hypothermia intraoperatively. The mean amount of intraoperative blood loss was $218.7 \pm 149.3 \mathrm{ml}$ (range $40-1050 \mathrm{ml}$ ). The incidence of perioperative transfusion was $13.4 \%(n=46)$. According to the univariate analysis, we determined the relationship between transfusion $(P=0.019)$ and hypothermia in the THA group (Table 4). The intraoperative temperature was negatively related to blood loss $(P=0.002)$ after adjustment in the multiple regression analysis. However, we did not find the relationship between the event of hypothermia and blood loss $(P=0.152)$ (Table 5). The multivariate logistic regression analysis indicated that hypothermia $(P=0.031, \mathrm{OR}=2.615)$ rather than intraoperative temperature $(P=0.073, \mathrm{OR}=0.122)$ was the independent risk factor for transfusion (Table 5). In the THA group, the hip fracture patients are different from the other patients in pathogenesis and clinical manifestation, which may influence the analysis. Therefore, we performed additional multivariate analysis without hip fracture patients. In these patients, we only found the negative relationship between intraoperative temperature and blood loss $(P=0.017)$ (data not shown).

\section{Discussion}

Inadvertent hypothermia occurs commonly during surgeries mainly due to skin exposure to cool environment, tissue loss, respiration, and disrupted thermoregulatory control caused 
TABLE 2: Univariate analysis of the risk factors for intraoperative hypothermia in TKA.

\begin{tabular}{|c|c|c|c|}
\hline Variable & No hypothermia $(\mathrm{n}=234)$ & Hypothermia $(\mathrm{n}=38)$ & $\mathrm{P}$ value \\
\hline Age $($ years $($ mean $\pm S D))$ & $68.1 \pm 7.2$ & $66.9 \pm 8.3$ & 0.326 \\
\hline Female gender (\%) & $193(82.5)$ & $29(76.3)$ & 0.363 \\
\hline BMI $\left(\mathrm{kg} / \mathrm{m}^{2}(\right.$ mean $\left.\pm \mathrm{SD})\right)$ & $26.3 \pm 3.8$ & $25.6 \pm 3.4$ & 0.268 \\
\hline Diabetes (\%) & $42(18.0)$ & $8(21.1)$ & 0.647 \\
\hline Hypertension (\%) & $108(46.2)$ & $14(36.8)$ & 0.284 \\
\hline Malignancy (\%) & $7(3.0)$ & $1(2.6)$ & 0.903 \\
\hline Diagnosis of RA (\%) & $21(9.0)$ & $5(13.2)$ & 0.416 \\
\hline Operation time $(\min ($ mean $\pm S D))$ & $123.4 \pm 23.4$ & $128.8 \pm 23.4$ & 0.185 \\
\hline $\mathrm{PT}(\mathrm{s}($ mean $\pm \mathrm{SD}))$ & $11.4 \pm 0.9$ & $11.3 \pm 1.1$ & 0.501 \\
\hline $\mathrm{INR}($ mean $\pm \mathrm{SD})$ & $0.99 \pm 0.08$ & $0.99 \pm 0.09$ & 0.786 \\
\hline APTT $(\mathrm{s}($ mean $\pm \mathrm{SD}))$ & $26.1 \pm 3.9$ & $25.5 \pm 3.6$ & 0.418 \\
\hline $\mathrm{Hb}(\mathrm{g} / \mathrm{l}($ mean $\pm \mathrm{SD}))$ & $126.0 \pm 13.1$ & $126.9 \pm 15.6$ & 0.706 \\
\hline $\operatorname{PLT}\left(10^{9} / 1(\right.$ mean $\left.\pm \mathrm{SD})\right)$ & $207.9 \pm 68.2$ & $200.4 \pm 75.6$ & 0.535 \\
\hline Blood loss $(\mathrm{ml}($ mean $\pm \mathrm{SD}))$ & $189.0 \pm 99.3$ & $239.5 \pm 116.9$ & $0.005 *$ \\
\hline Transfusion (\%) & $33(14.1)$ & $15(39.5)$ & $<0.001 *$ \\
\hline
\end{tabular}

${ }^{*} P<0.05$ was considered statistically significant. SD: standard deviation; BMI: body mass index; RA: rheumatoid arthritis; PT: prothrombin time; INR: international normalized ratio; APTT: activated partial thromboplastin time; Hb: hemoglobin; PLT: platelet count.

TABLE 3: The association of intraoperative temperature and hypothermia with intraoperative blood loss and perioperative blood transfusion in TKA.

\begin{tabular}{lcccc}
\hline & Intraoperative blood loss & & \multicolumn{2}{c}{ Perioperative blood transfusion } \\
& Unstandardized coefficients & P value & OR & 0.080 \\
\hline Intraoperative temperature & -62.700 & $0.045 *$ & $0.006 *$ \\
Hypothermia & 35.974 & $0.042 *$ & 4.468 & $<0.001 *$ \\
\hline
\end{tabular}

${ }^{*} P<0.05$ was considered statistically significant. OR: odds ratio.

TABLE 4: Univariate analysis of the risk factors for intraoperative hypothermia in THA.

\begin{tabular}{|c|c|c|c|}
\hline Variable & No hypothermia $(\mathrm{n}=299)$ & Hypothermia $(\mathrm{n}=45)$ & $\mathrm{P}$ value \\
\hline Age (years $($ mean $\pm S D))$ & $64.0 \pm 14.3$ & $65.9 \pm 12.5$ & 0.397 \\
\hline Female gender (\%) & $173(57.9)$ & $31(68.9)$ & 0.160 \\
\hline $\mathrm{BMI}\left(\mathrm{kg} / \mathrm{m}^{2}(\right.$ mean $\left.\pm \mathrm{SD})\right)$ & $23.5 \pm 3.6$ & $23.9 \pm 3.6$ & 0.539 \\
\hline Diabetes (\%) & $28(9.4)$ & $7(15.6)$ & 0.200 \\
\hline Hypertension (\%) & $95(31.8)$ & $20(44.4)$ & 0.093 \\
\hline Malignancy (\%) & $7(2.3)$ & 0 & 0.300 \\
\hline Diagnosis & - & - & 0.377 \\
\hline Operation time $($ min $($ mean $\pm S D))$ & $101.7 \pm 27.4$ & $105.6 \pm 29.2$ & 0.372 \\
\hline $\mathrm{PT}(\mathrm{s}($ mean $\pm \mathrm{SD}))$ & $11.6 \pm 0.9$ & $11.6 \pm 1.2$ & 0.890 \\
\hline $\mathrm{INR}($ mean $\pm \mathrm{SD})$ & $1.02 \pm 0.08$ & $1.01 \pm 0.11$ & 0.623 \\
\hline APTT $(\mathrm{s}($ mean \pm SD) $)$ & $27.5 \pm 4.8$ & $27.9 \pm 5.6$ & 0.656 \\
\hline $\mathrm{Hb}(\mathrm{g} / \mathrm{l}($ mean $\pm \mathrm{SD}))$ & $129.2 \pm 15.3$ & $128.0 \pm 14.9$ & 0.629 \\
\hline $\operatorname{PLT}\left(10^{9} / 1(\right.$ mean $\left.\pm \mathrm{SD})\right)$ & $201.1 \pm 64.9$ & $194.5 \pm 62.2$ & 0.522 \\
\hline Blood loss $(\mathrm{ml}($ mean $\pm \mathrm{SD}))$ & $214.5 \pm 137.3$ & $246.7 \pm 212.2$ & 0.178 \\
\hline Transfusion (\%) & $35(11.7)$ & $11(24.4)$ & $0.019 *$ \\
\hline
\end{tabular}

${ }^{*} P<0.05$ was considered statistically significant. SD: standard deviation; BMI: body mass index; PT: prothrombin time; INR: international normalized ratio; APTT: activated partial thromboplastin time; Hb: hemoglobin; PLT: platelet count. 
TABLE 5: The association of intraoperative temperature and hypothermia with intraoperative blood loss and perioperative blood transfusion in THA.

\begin{tabular}{lcccc}
\hline & Intraoperative blood loss & \multicolumn{2}{c}{ Perioperative blood transfusion } \\
& Unstandardized coefficients & P value & \multicolumn{2}{c}{ OR value } \\
\hline Intraoperative temperature & -153.067 & $0.002 *$ & 0.122 & 0.073 \\
Hypothermia & 30.138 & 0.152 & 2.615 & $0.031 *$ \\
\hline
\end{tabular}

${ }^{*} P<0.05$ was considered statistically significant. OR: odds ratio.

by general anesthesia [15]. In this study, we identified the incidence of intraoperative hypothermia in TJA and attempted to explore the risk factors. The rates of intraoperative hypothermia were $13.5 \%, 14.0 \%$, and $13.1 \%$ in TJA, TKA and THA, respectively. The incidence of hypothermia reported by previous studies varied widely from $11.2 \%$ to 43.9\% [11-13], which may be caused by different warming methods, anesthesia procedure, and operating room environment. According to previous studies, female gender, lower BMI, knee arthroplasty, neuraxial anesthesia, lower preoperative temperature, lower operating room temperature, and longer operation time are the risk factors for hypothermia in TJA $[11,13,16]$. Therefore, measures improving the control of modifiable risk factors may be able to reduce the rate of hypothermia.

By employing multiple regression analysis and multivariate logistic regression analysis, intraoperative temperature and hypothermia were found to be independently associated with intraoperative blood loss and perioperative transfusion in TKA. In THA, intraoperative temperature was negatively related to the amount of blood loss, and hypothermia was the independent risk factor for transfusion. Rajagopalan et al. conducted a systematic review to evaluate the effects of hypothermia on surgical patients and found that even mild hypothermia increased blood loss and risk for transfusion [17]. Schmied et al. found that mild hypothermia increased transfusion requirements and augmented blood loss by approximately $500 \mathrm{ml}$ in patients undergoing THA [18]. Frisch et al. investigated the impact of intraoperative hypothermia in TKA and THA and also found that hypothermia was associated with increased blood loss. However, the relationship between hypothermia and blood transfusion was not observed in their study [13]. The impaired coagulation system induced by hypothermia may explain the increased blood loss. Hypothermia has been proven to impair the coagulation cascade by inhibiting enzymatic reactions, which causes prolonged prothrombin time and partial thromboplastin time [19]. Mild hypothermia also reduces the adhesion ability of the platelet and the subsequent coagulation process [20]. Besides, hypothermia has been demonstrated to inhibit platelet activation in vitro and in vivo, which slows down the formation of a fibrin-rich hemostatic plug [21].

Intraoperative hypothermia also increases the risk of reoperation and postoperative infection in TJA [11]. Therefore, maintenance of normothermia may decrease the risk of postoperative complications and improve outcomes. Warming strategies are classified into passive and active warming systems. Passive warming systems refer to the layers of insulation, such as blankets and surgical drapes. Active warming systems include forced air, circulating water, radiant warmer, fluid warming, and airway heating [15]. For patients undergoing surgeries, the factor that contributes most to perioperative hypothermia is the internal redistribution of body heat caused by general or neuraxial anesthesia [22]. Therefore, it is important to monitor temperature and maintain normothermia in this population. Another reason for inadvertent hypothermia is the exposure of the skin to the environment of the operation room. Therefore, efforts should be made to maintain an optimal operation room temperature, which can reduce the risk of complications [23]. Body temperature has been reported to drop obviously between preoperative holding and induction of anesthesia [12]. A previous study used preoperative forced-air warming and found that it could significantly decrease the rate of intraoperative hypothermia [24].

There are several limitations in this study. First, the present study has all the inherent limitations of a retrospective study. Second, the "intraoperative temperature," which we used for analysis, was calculated by taking the average of temperature readings from an esophageal detector. Since the readings were recorded at intervals of 5 minutes, the "intraoperative temperature" could not completely represent the temperature during the entire process of surgery. Third, the amount of intraoperative blood loss was estimated by the volume of blood in the suction canister plus that in the sponges in this study. More precise amount of blood loss should be evaluated by formulas based on laboratory data, such as Gross' equation. Fourth, larger sample size and more confounders should be used to explore the risk factors for hypothermia. Besides, we did not use active warming devices routinely. Forced air warming would be employed when the temperature dropped to $36^{\circ} \mathrm{C}$. However, we did not collect the exact number of patients receiving forced air warming, which may affect the analysis.

In conclusion, intraoperative temperature and hypothermia were associated with intraoperative blood loss and perioperative transfusion rates in TKA. Intraoperative temperature was negatively related to the amount of blood loss, and hypothermia was the independent risk factor for transfusion in THA. Therefore, strategies to maintain normothermia should be employed to reduce blood loss and transfusion rates in these patients.

\section{Data Availability}

The data used to support the findings of this study are available from the corresponding author upon request. 


\section{Conflicts of Interest}

All authors declared that they have no conflicts of interest to this work.

\section{Authors' Contributions}

Pin Pan and Kai Song contributed equally to this research and share joint first authorship.

\section{Acknowledgments}

This work was supported by the Jiangsu Provincial Key Research and Development Foundation (BE2016608), National Science Foundation of China (81702151), Natural Science Foundation of Jiangsu Province (BK20170121), and Fundamental Research Funds for the Central Universities (021414380306).

\section{References}

[1] J. F. Maempel, N. R. Wickramasinghe, N. D. Clement, I. J. Brenkel, and P. J. Walmsley, "The pre-operative levels of haemoglobin in the blood can be used to predict the risk of allogenic blood transfusion after total knee arthroplasty," The Bone \& Joint Journal, vol. 98-B, no. 4, pp. 490-497, 2016.

[2] J. A. Salido, L. A. Marín, L. A. Gómez, P. Zorrilla, and C. Martínez, "Preoperative hemoglobin levels and the need for transfusion after prosthetic hip and knee surgery: analysis of predictive factors," The Journal of Bone and Joint Surgery. American Volume, vol. 84, no. 2, pp. 216-220, 2002.

[3] B. Horosz and M. Malec-Milewska, "Metody przeciwdziałania śródoperacyjnej hipotermii," Anestezjologia Intensywna Terapia, vol. 46, no. 2, pp. 96-100, 2014.

[4] H. K. Lee, Y. H. Jang, K. W. Choi, and J. H. Lee, "The effect of electrically heated humidifier on the body temperature and blood loss in spinal surgery under general anesthesia," Korean Journal of Anesthesiology, vol. 61, no. 2, pp. 112-116, 2011.

[5] T. R. Jildeh, K. R. Okoroha, N. E. Marshall et al., "The effect of intraoperative hypothermia on shoulder arthroplasty," Orthopedics, vol. 41, no. 4, pp. e523-e528, 2018.

[6] M. D. Schur, G. W. Blumstein, D. A. Seehausen, P. A. Ross, L. M. Andras, and D. L. Skaggs, "Intraoperative hypothermia is common, but not associated with blood loss or transfusion in pediatric posterior spinal fusion," Journal of Pediatric Orthopaedics, vol. 38, no. 9, pp. 450-454, 2018.

[7] N. S. Tedesco, F. P. Korpi, V. K. Pazdernik, and J. M. Cochran, "Relationship between hypothermia and blood loss in adult patients undergoing open lumbar spine surgery," The Journal of the American Osteopathic Association, vol. 114, no. 11, pp. 828-838, 2014.

[8] B. Leijtens, M. Koeter, K. Kremers, and S. Koeter, "High incidence of postoperative hypothermia in total knee and total hip arthroplasty: a prospective observational study," The Journal of Arthroplasty, vol. 28, no. 6, pp. 895-898, 2013.

[9] S. Brandt, R. Oguz, H. Hüttner et al., "Resistive-polymer versus forced-air warming: comparable efficacy in orthopedic patients," Anesthesia and Analgesia, vol. 110, no. 3, pp. 834838, 2010.

[10] M. F. Sandoval, P. D. Mongan, M. R. Dayton, and C. A. Hogan, "Safety and efficacy of resistive polymer versus forced air warming in total joint surgery," Patient Safety in Surgery, vol. 11, p. 11, 2017.

[11] M. Williams and Y. El-Houdiri, "Inadvertent hypothermia in hip and knee total joint arthroplasty," Journal of Orthopaedics, vol. 15, no. 1, pp. 151-158, 2018.

[12] J. B. Simpson, V. S. Thomas, S. K. Ismaily, P. I. Muradov, P. C. Noble, and S. J. Incavo, "Hypothermia in total joint arthroplasty: a wake-up call," The Journal of Arthroplasty, vol. 33, no. 4, pp. 1012-1018, 2018.

[13] N. B. Frisch, A. M. Pepper, E. Rooney, and C. Silverton, "Intraoperative hypothermia in total hip and knee arthroplasty," Orthopedics, vol. 40, no. 1, pp. 56-63, 2017.

[14] M. Dan, S. M. Martos, E. Beller, P. Jones, R. Randle, and D. Liu, "Blood loss in primary total knee arthroplasty-body temperature is not a significant risk factor-a prospective, consecutive, observational cohort study," Journal of Orthopaedic Surgery and Research, vol. 10, p. 97, 2015.

[15] M. W. Allen and D. J. Jacofsky, "Normothermia in arthroplasty," The Journal of Arthroplasty, vol. 32, no. 7, pp. 23072314, 2017.

[16] J. R. Matos, J. R. McSwain, B. J. Wolf, J. W. Doty, and S. H. Wilson, "Examination of intra-operative core temperature in joint arthroplasty: a single-institution prospective observational study," International Orthopaedics, vol. 42, no. 11, pp. 2513-2519, 2018.

[17] S. Rajagopalan, E. Mascha, J. Na, and D. I. Sessler, "The effects of mild perioperative hypothermia on blood loss and transfusion requirement," Anesthesiology, vol. 108, no. 1, pp. 71-77, 2008.

[18] H. Schmied, A. Kurz, D. I. Sessler, S. Kozek, and A. Reiter, "Mild hypothermia increases blood loss and transfusion requirements during total hip arthroplasty," Lancet, vol. 347, no. 8997, pp. 289-292, 1996.

[19] M. J. Rohrer and A. M. Natale, "Effect of hypothermia on the coagulation cascade," Critical Care Medicine, vol. 20, no. 10, pp. 1402-1405, 1992.

[20] A. S. Wolberg, Z. H. Meng, D. M. Monroe III, and M. Hoffman, "A systematic evaluation of the effect of temperature on coagulation enzyme activity and platelet function," The Journal of Trauma, vol. 56, no. 6, pp. 1221-1228, 2004.

[21] A. D. Michelson, H. MacGregor, M. R. Barnard, A. S. Kestin, M. J. Rohrer, and C. R. Valeri, "Reversible inhibition of human platelet activation by hypothermia in vivo and in vitro," Thrombosis and Haemostasis, vol. 71, no. 5, pp. 633-640, 1994.

[22] D. I. Sessler, "Perioperative thermoregulation and heat balance," Lancet, vol. 387, no. 10038, pp. 2655-2664, 2016.

[23] A. Aalirezaie, M. Akkaya, C. L. Barnes et al., "General assembly, prevention, operating room environment: proceedings of international consensus on orthopedic infections," The Journal of Arthroplasty, vol. 34, no. 2, pp. S105-S115, 2019.

[24] A. B. Kay, D. M. Klavas, T. Hirase, M. O. Cotton, B. S. Lambert, and S. J. Incavo, "Preoperative warming reduces intraoperative hypothermia in total joint arthroplasty patients," The Journal of the American Academy of Orthopaedic Surgeons, vol. 28, no. 6, pp. e255-e262, 2020. 